JOURNAL OF SYNCHROTRON RADIATION

ISSN 1600-5775

Received 12 February 2016

Accepted 14 February 2016

Keywords: detectors; hybrid pixel array detectors; HPADs; low-noise detectors.

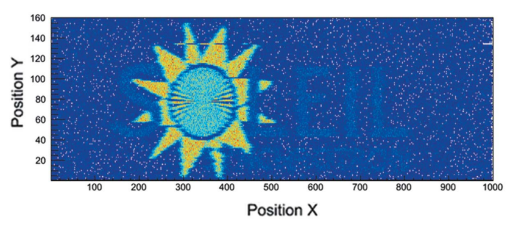

(C) 2016 International Union of Crystallography

\section{Hybrid pixel array detectors enter the low noise regime}

\author{
H. Graafsma*
}

Center for Free-Electron Laser Science, DESY, 22607 Hamburg, Germany, and Mid Sweden University, Sundsvall, Sweden. *Correspondence e-mail: heinz.graafsma@desy.de

The detector group at the Swiss Light Source (SLS) at PSI has been one of the pioneers and driving forces for the development of custom-made hybrid pixel array detectors (HPADs) for synchrotron applications. In their paper Jungmann-Smith et al. (2016) show that it is now possible to develop HPADs with sufficiently low noise to allow singlephoton detection below $1 \mathrm{keV}$ as well as to perform spectroscopic imaging.

For decades detectors have been a limiting factor in experiments at synchrotron radiation facilities. Even though imaging detectors evolved over time, from photographic films, via gas-filled detectors and imaging plates to CCD-based systems, the evolution of the source always outran the evolution of the detector. One of the reasons was the fact that detectors were mainly constructed by adapting systems or components developed for other applications, like astronomy or medical imaging, as best as possible to the scientific needs at the experimental stations. This situation started to change with the introduction of the so-called hybrid pixel array detectors (Rossi et al., 1999; Broennimann et al., 2006). At the heart of these detectors, pioneered by the high-energy physics community, is an application-specific integrated circuit (ASIC), which is a pixelated readout chip customdesigned for a well defined experiment or technique. One of the revolutionizing advantages offered by this technology is that every single pixel contains all necessary electronics, including for instance counters, for X-ray detection. This massive parallelization increased the overall efficiency of the detector by several orders of magnitude as compared with the CCD-based system. There are now various examples of hybrid pixel array detectors, specifically developed for X-ray experiments at storage ring synchrotron sources (Hatsui \& Graafsma, 2015), as well as various spin-off companies commercializing them. Most of these systems are so-called photon-counting detectors, where each incoming photon is processed by the readout electronics in the pixel and individually counted. The advantage of photon counting is that electronic noise, present in any system, can be efficiently discriminated against, yielding 'noise-free' detectors.

An enormous boost of the development of HPADs is provided by X-ray free-electron lasers due to the extreme requirements imposed by these new sources (Graafsma, 2009). New dedicated readout circuitry, with entirely different concepts, had to be designed. For one thing, photon-counting techniques cannot be employed since too many photons arrive at the same time, e.g. up to $10^{5}$ per pulse per pixel in the low-angle region for some experiments. Therefore, detector developers resorted back to integrating systems. In integrating detectors the total number of electrons (or holes) created in the sensor by the absorbed photons are integrated during the exposure time. This makes the detectors extremely fast. All photons can arrive at the same time, since the electronics does not need to count them. As a result, integrating HPAD systems are considered an excellent choice for fast experiments with high intensities. However, up until now, integrating HPADs were considered less suited for low intensity, low noise or spectroscopic imaging.

With the JUNGFRAU 0.4 prototype system described by Jungmann-Smith et al. (2016), the detector group at the PSI/SLS has opened up yet another application field for HPADs. The noise of the system is with 27 electrons extremely low for an HPAD. 27 electrons is the amount of electron-hole pairs created in silicon by a $100 \mathrm{eV}$ photon. This means that the JUNGFRAU 0.4 system is able to detect $0.5 \mathrm{keV}$ photons with a signal-to-noise ratio of 5, making it an interesting system for many soft X-ray freeelectron stations. The very limited dynamic range of the JUNGFRAU 0.4 could be 

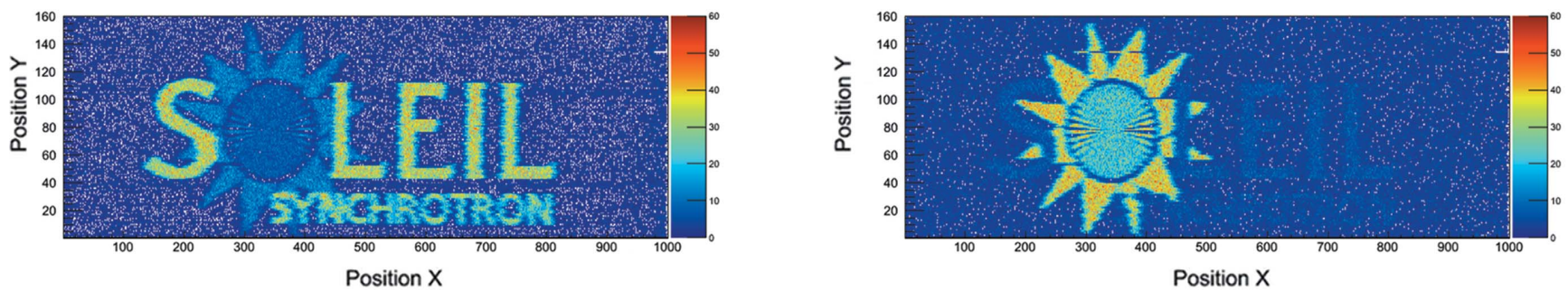

Figure 1

Fly-scan images of the SOLEIL logo taken with the JUNGFRAU 0.4. The letters of SOLEIL are made of Ni, while the material of the sun (with a Siemens resolution star at the centre) is Au. By selecting the corresponding energy windows in the data analysis routines the two materials are clearly separated, albeit with some remaining cross talk.

overcome by implementing adaptive-gain amplifiers, similar to what is done in the standard JUNGFRAU and AGIPD detectors (Mozzanica et al., 2014; Henrich et al., 2011). Another application for such a low noise system is for energydispersive measurements. Jungmann-Smith et al. (2016) show in their paper that, with the use of a proper mask to shield the edge regions between pixels, very good fluorescence spectra can be obtained. This capability was subsequently used for multi-colour imaging at the SOLEIL synchrotron using the flyscan technique. The composition of a SOLEIL logo $(\mathrm{Ni}, \mathrm{Au})$ was easily determined with high resolution (Fig. 1). JungmannSmith et al. (2016) introduce and compare different techniques and algorithms to obtain the best possible energy resolution.

The innovative aspect of this paper does not lie in the spectroscopic results obtained since they could very well have been obtained with other systems, like silicon drift detectors (SDDs) or the Maia detector (Siddons et al., 2014) for instance. But what is truly impressive is that these results were obtained with a hybrid pixel array detector using a standard planar diode array as sensor. This means that the system uses relatively standard and thus easy to manufacture components, making it possible to envision building larger and/or further optimized systems in the near future. And with that, low-noise HPADs have entered a field formally reserved for SSDs and CMOS imagers.

\section{References}

Broennimann, Ch., Eikenberry, E. F., Henrich, B., Horisberger, R., Huelsen, G., Pohl, E., Schmitt, B., Schulze-Briese, C., Suzuki, M., Tomizaki, T., Toyokawa, H. \& Wagner, A. (2006). J. Synchrotron Rad. 13, 120-130.

Graafsma, H. (2009). J. Instrum. 4, P12011.

Hatsui, T. \& Graafsma, H. (2015). IUCrJ, 2, 371-383.

Henrich, B., Becker, J., Dinapoli, R., Goettlicher, P., Graafsma, H., Hirsemann, H., Klanner, R., Krueger, H., Mazzocco, R., Mozzanica, A., Perrey, H., Potdevin, G., Schmitt, B., Shi, X., Srivastava, A. K., Trunk, U. \& Youngman, C. (2011). Nucl. Instrum. Methods Phys. Res. A, 633, S11-S14.

Jungmann-Smith, J. H., Bergamaschi, A., Brückner, M., Cartier, S., Dinapoli, R., Greiffenberg, D., Huthwelker, T., Maliakal, D., Mayilyan, D., Medjoubi, K., Mezza, D., Mozzanica, A., Ramilli, M., Ruder, Ch., Schädler, L., Schmitt, B., Shi, X. \& Tinti, G. (2016). J. Synchrotron Rad. 23, 385-394.

Mozzanica, A., Bergamaschi, A., Cartier, S., Dinapoli, R., Greiffenberg, D., Johnson, I., Jungmann, J., Maliakal, D., Mezza, D., Ruder, C., Schaedler, L., Schmitt, B., Shi, X. \& Tinti, G. (2014). J. Instrum. 9, C05010.

Rossi, G., Renzi, M., Eikenberry, E. F., Tate, M. W., Bilderback, D., Fontes, E., Wixted, R., Barna, S. \& Gruner, S. M. (1999). J. Synchrotron Rad. 6, 1096-1105.

Siddons, D. P., Kirkham, R., Ryan, C. G., De Geronimo, G., Dragone, A., Kuczewski, A. J., Li, Z. Y., Carini, G. A., Pinelli, D., Beuttenmuller, R., Elliott, D., Pfeffer, M., Tyson, T. A., Moorhead, G. F. \& Dunn, P. A. (2014). J. Phys. Conf. Ser. 499, 012001 . 